\title{
Sutura hemostática uterina en asociación con taponamiento intrauterino con balón SOS Bakri ("sandwich uterino") para hemorragia posparto: reporte de un caso.
}

\author{
María Saldari, ${ }^{1}$ (D) Jesús Alejandro Veroes, ${ }^{2}$ (D) María Martínez, ${ }^{1}$ (D) Vanessa Yelamo, ${ }^{1}$ \\ Marielys Colmenares. ${ }^{2}$
}

\begin{abstract}
RESUMEN
La hemorragia posparto es la principal causa de morbimortalidad materna en todo el mundo. La rapidez con la que se presenta, en conjunto con la imprecisión clínica para estimar la pérdida sanguínea requiere una atención expedita. Se presenta el caso de una paciente que consultó en el Hospital Luis Ortega de Porlamar, Venezuela, por sangrado uterino persistente, secundario a atonía uterina, resistente a la administración de oxitócicos. Se efectuó sutura hemostática B-Lynch, sin lograr hemostasia, por lo que subsecuentemente se procede a realizar taponamiento uterino con balón SOS Bakri, obteniendo resultados satisfactorios. Los hallazgos se comparan con los datos globales reportados en otras publicaciones.
\end{abstract}

Palabras clave: Hemorragia posparto, Atonía uterina, Sutura hemostática, Taponamiento uterino, Sándwich uterino.

\section{SUMMARY}

Postpartum hemorrhage is the leading cause of maternal morbidity and mortality worldwide. The rapidity with which it can occur along with the notorious clinical imprecision in estimates of blood loss requires prompt management of care. We present the case of a patient who consulted at the Luis Ortega Hospital in Porlamar, Venezuela, due to persistent uterine bleeding, secondary to uterine atony resistant to the administration of oxytocic. B-Lynch hemostatic suture was performed without achieving hemostasis, for which reason uterine tamponade with SOS Bakri balloon was subsequently performed, obtaining satisfactory results. The findings are compared with the global data reported in other publications.

Keywords: Postpartum hemorrhage, Uterine atony, Hemostatic suture, Uterine tamponade, Uterine sandwich

\section{INTRODUCCIÓN}

La hemorragia posparto (HPP) es una de las tres primeras causas de mortalidad materna en el mundo, en especial en países en vías de desarrollo. Aunque son múltiples las definiciones empleadas para la HPP, la más aceptada actualmente, es la pérdida de cualquier cantidad de sangre que cause signos de hipovolemia y/o inestabilidad hemodinámica en la paciente (1).

'Especialista Ginecología y Obstetricia Hospital Luis Ortega Porlamar, Nueva Esparta, Venezuela. ${ }^{2}$ Colaborador Docente Ginecología y Obstetricia Hospital Luis Ortega Porlamar, Nueva Esparta, Venezuela.
Hasta en un $80 \%$ de los casos, la etiología de la HPP se relaciona con la atonía uterina, secundaria a hipocontractilidad disfuncional del miometrio durante el puerperio inmediato (2).

Para el tratamiento de la HPP, se deben tomar en cuenta los siguientes aspectos: la toma de decisiones por un equipo multidisciplinario, la etiología del sangrado, las opciones terapéuticas disponibles y el deseo de fertilidad futura, con el objetivo de controlar el sangrado y lograr la hemostasia. Se inicia con masaje uterino bimanual, acompañado de la administración de uterotónicos (oxitocina, metilergonovina, prostaglandinas sintéticas) y hemostáticos; en caso de 
continuar la pérdida hemática se recurre al tratamiento conservador que incluye desde el taponamiento uterino, la ligadura de las arterias hipogástricas, uterinas $\mathrm{u}$ ováricas, las suturas compresivas tipo B-Lynch, hasta la embolización de arterias uterinas y/o hipogástricas y, como última opción, la histerectomía (3).

La tendencia actual es no utilizar protocolos prestablecidos, sino la individualización de cada caso, de tal forma que la secuencia de técnicas a implementar no siempre será la misma. Las suturas de compresión uterina tipo B-Lynch y los balones hemostáticos intrauterinos (tipo SOS Bakri) se proponen como tratamiento conservador en los casos de HPP. Estas estrategias quirúrgicas se pueden utilizar solas o en combinación ("sándwich uterino"), para aplicar tensión sinérgica a ambas superficies del miometrio resultando exitosa en los casos de atonía uterina persistente y hemorragia masiva (Figura 1) (4-6).

Se presenta el caso de una paciente con HPP por atonía uterina secundaria a desprendimiento prematuro de placenta normoinserta, con falla en la respuesta a uterotónicos y a quien se le aplicó la técnica de sándwich uterino.

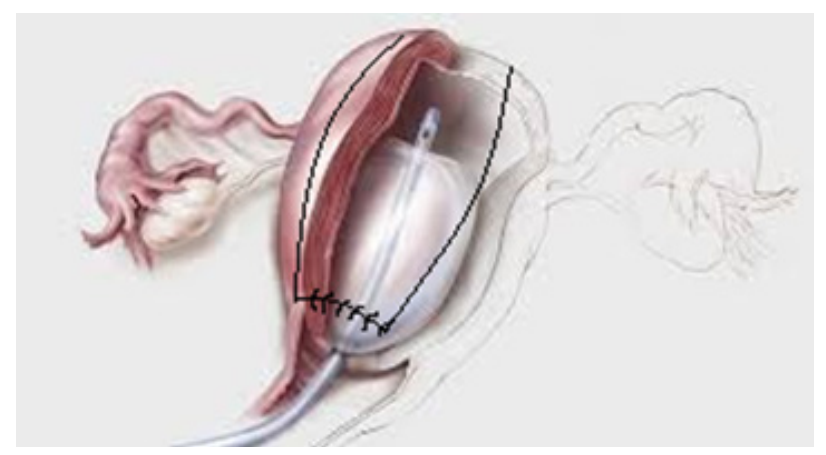

Figura 1. Representación esquemática del "sándwich uterino"

\section{RESUMEN DE LA HISTORIA CLÍNICA}

Paciente de 29 años de edad, II G I P, con gestación de 34 semanas, acudió a consulta prenatal en 5 oportunidades, sin antecedentes personales o quirúrgicos de relevancia, quien consultó a la emergencia obstétrica del Hospital Luis Ortega de Porlamar por presentar sangrado a través de genitales externos, con coágulos, en moderada cantidad. Concomitantemente, refería dolor en hipogastrio, tipo punzante, de fuerte intensidad, sin atenuantes. Al examen físico se encontró: signos vitales: presión arterial 95/60 mmHg, frecuencia cardíaca: 100 latidos por minuto, frecuencia respiratoria: 19 por minuto; palidez cutáneo mucosa acentuada. Abdomen gestante, feto único, presentación cefálica, frecuencia cardiaca fetal: 118 latidos por minuto, útero hipertónico. Al espéculo se evidenció el cuello corto, permeable, y abundante sangrado con coágulos. Se ingresó con los siguientes diagnósticos: 1. Embarazo simple de 34 semanas 2. Desprendimiento prematuro de placenta normoinserta.

Los resultados de los exámenes de laboratorio de ingreso fueron los siguientes: hemoglobina $11,5 \mathrm{~g} /$ dL; hematocrito: 34 vol \%; plaquetas: 160 000; VIH negativo; VDRL no reactivo; tiempo de protrombina (PT): 13 segundos, control 13,48 segundos; tiempo parcial de tromboplastina (PTT): 30 segundos, control 31,5 segundos.

Se realizó cesárea segmentaria, bajo anestesia general, incisión tipo pfannenstiel, evidenciando posterior a la histerotomía, presencia de abundante sangrado con coágulos en cavidad amniótica, se obtuvo recién nacido pretérmino, masculino, con apgar de 4 y 7 puntos al nacer y al minuto, respectivamente, que pesó 2180 gramos. Se realizó extracción manual de la placenta y se observaron signos de desprendimiento de aproximadamente $70 \%$; las caras anterior, posterior y el fondo uterino tenían un infiltrado difuso, sin 
tono, a pesar de la administración de oxitócicos. Se realizó sutura hemostática de B-Lynch, con técnica clásica, utilizando catgut crómico 1 , histerorrafia con catgut crómico 0 , con comprobación de la hemostasia (Figura 2). Se realizó la síntesis de pared abdominal por planos.

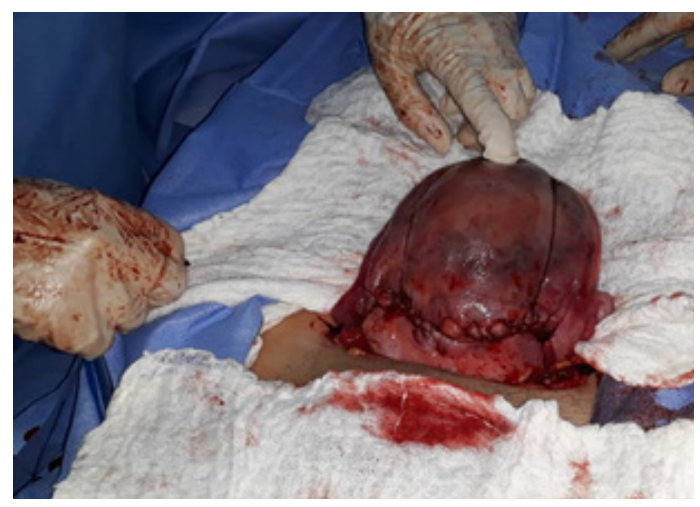

Figura 2. Colocación de sutura hemostática

La paciente se trasladó al área de recuperación para monitorización. A los 13 minutos presentó sangrado profuso a través de los genitales con signos de inestabilidad hemodinámica. Por no contar con otros uterotónicos, hemostáticos, ni hemoderivados, se decidió colocación de taponamiento uterino con balón SOS Bakri, que concluyó con llenado con 400 cc de solución 0,9 \% y altura uterina: $23 \mathrm{~cm}$ (Figura $3)$. Se colocó compresa en el canal vaginal. Se evidenció mejoría de estado hemodinámico posterior al procedimiento. La paciente fue trasladada al área de preparto en condiciones clínicas estables, se mantuvo en observación con registro horario de signos vitales, diuresis, altura uterina y gasto hemático a través del balón. El control paraclínico (tomado durante el episodio de hemorragia) reportó: hemoglobina 9,9 g/ dL; hematocrito: $31 \%$; leucocitos 20 100; plaquetas 258 000; creatinina 0,5 mg/dL; glicemia $94 \mathrm{mg} / \mathrm{dL}$; urea $29 \mathrm{mg} / \mathrm{dL}$; PT 13 segundos, control 14 segundos; PTT 30 segundos, control 30 segundos. A las 8 horas postaponamiento se evidenció reducción de la altura uterina en $17 \mathrm{~cm}$, con evolución clínica y paraclínica

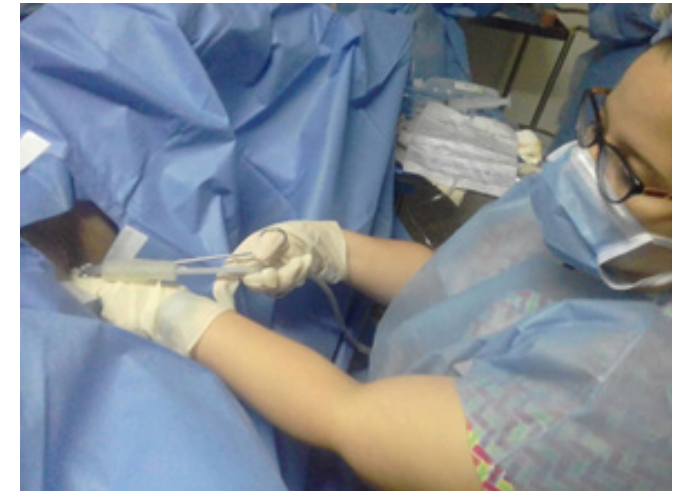

Figura 3. Colocación de taponamiento uterino

satisfactoria, por lo que se inició protocolo de retiro progresivo que duró 4 horas. La revisión del canal vaginal mostró que estaba indemne con sangrado escaso. El gasto total cuantificado a través del balón SOS Bakri durante las 12 horas que estuvo colocado fue de $105 \mathrm{~mL}$. El control paraclínico (tomado a las 6 horas de la estabilización hemodinámica): hemoglobina $8 \mathrm{~g} / \mathrm{dL}$; hematocrito $25 \%$; leucocitos 12 700; plaquetas 240 000. Permaneció hospitalizada para optimizar las cifras de hemoglobina, luego de 6 días egresó en buenas condiciones clínicas generales. Se realizó control ultrasonográfico a los 6 meses y se evidenció normalidad endometrial.

\section{DISCUSIÓN}

Se denomina sándwich uterino a la combinación de realización de una sutura hemostática acompañada de la colocación de un taponamiento uterino, en el contexto de una HPP que no responde a uterotónicos. El mecanismo de acción pudiese explicarse debido a la fuerza de tensión externa ofrecida por la sutura hemostática asociada con la presión intrauterina aportada por el taponamiento, aplicando un efecto compresivo a ambas superficies del miometrio, lo cual favorece a la disminución del sangrado (4). 


\section{SUTURA HEMOSTÁTICA UTERINA EN ASOCIACIÓN CON TAPONAMIENTO INTRAUTERINO CON BALÓN SOS BAKRI ("SANDWICH UTERINO") PARA HEMORRAGIA POSPARTO: REPORTE DE UN CASO.}

Yoong y cols. (4) presentaron una serie de 11 casos donde colocaron suturas hemostáticas de B-Lynch, en sus dos primeros casos, y con la modificación de Haymann, en los nueve siguientes, todos acompañados de taponamiento uterino con balón SOS Bakri. Nelson y cols, (5) presentaron 5 pacientes que recibieron tratamiento, con balón SOS Bakri en conjunto con la sutura de compresión uterina B-Lynch, con éxito en el tratamiento de atonía uterina. En el presente caso se utilizó como sutura hemostática la técnica clásica de B-Lynch.

Revisiones anteriores (4, 6), documentaron la aplicación de la técnica de sándwich uterino en pacientes con diagnóstico de placenta previa como factor de riesgo para HPP. Price y cols. (7) aplicaron la sutura del mismo nombre con la asociación intrauterina de un catéter con balón para hemorragia masiva debido a una placenta ácreta después de un aborto. El caso presentado se trata de un desprendimiento prematuro de placenta normoinserta en el cual se evidenció infiltración del miometrio como causa de la atonía uterina.

Respecto al material de sutura utilizado, Nelson y cols. (5) confeccionaron su técnica hemostática con poliglactina $910 \mathrm{~N}^{\circ} 1$. Price y cols. (7), utilizaron monocryl y la recomendaron como ideal para la realización de la técnica, en contraste con el caso presente en el que se utilizó catgut crómico $\mathrm{N}^{\mathrm{o}} 1$, por ser el de disponibilidad en la institución.

La altura uterina fue un parámetro clínico que se utilizó para descartar la acumulación de sangre entre el balón y la pared uterina así como un signo de recuperación de la contractilidad miometrial. Rodríguez y cols. (3), en su investigación acerca de taponamiento uterino no asociado a sutura hemostática, describieron la tendencia al descenso de la altura uterina, probablemente debido a la contracción del miometrio en el momento de recuperación del episodio de atonía, los autores encontraron valores de $24 \pm 2 \mathrm{~cm}$ durante la atonía uterina y $22 \pm 3 \mathrm{~cm}$ después de colocar el balón. Este parámetro se registró en este caso, obteniendo medidas de 23 y $17 \mathrm{~cm}$ respectivamente. Se debe destacar que la variación en las mediciones probablemente estuvo determinada por la combinación con una sutura hemostática en contraste con el estudio antes mencionado en el cual se realizó solo el taponamiento uterino.

En la serie de Nelson y cols. (5), el balón intrauterino estuvo en su lugar durante una media de 11 horas (rango 10-24 horas), mientras que Yoong y cols. (4) reportaron una media de 22 horas (rango 17-27 horas). En el caso presentado, se logró evidenciar signos clínicos y paraclínicos favorables a las 8 horas, iniciándose el protocolo de retiro en ese lapso, el cual se concluyó en 4 horas, para completar un tiempo total de duración del taponamiento uterino de 12 horas.

En cuanto al volumen de llenado del balón SOS Bakri, Yoong y cols. (4) usaron un volumen medio de inflado de $300 \mathrm{~mL}$ (rango $150-350 \mathrm{~mL}$ ). El volumen medio infundido por Nelson y cols. (5) en el balón fue de $100 \mathrm{~mL}$ (rango 60-250 mL). En el presente caso se empleó un volumen de llenado de $400 \mathrm{~mL}$; en lugar del volumen de $500 \mathrm{~mL}$ sugerido por el fabricante; destacando que dicha recomendación es para taponamientos no asociados a suturas hemostáticas. La realización del B-Lynch, en combinación con el taponamiento es un aspecto importante para disminuir el volumen de insuflación del balón recomendado.

Nelson y cols. (5) reportaron, en su serie, una pérdida de sangre estimada de $2500 \mathrm{~mL}$ (rango $2000 \mathrm{~mL}-3000$ $\mathrm{mL}$ ), mientras que Yoong y cols. (4) la estimaron en promedio en $1500 \mathrm{~mL}$ (rango 750-4000 ml). En el presente caso se cuantificó una pérdida intraoperatoria de $1250 \mathrm{~mL}$ y durante el episodio de atonía uterina en sala de recuperación aproximadamente $1500 \mathrm{~mL}$, para un total de $2750 \mathrm{~mL}$. 
El estudio realizado de Nelson y cols. (5) reportó una mediana del hematocrito del 21,1 \% (rango 20,1 vol \% a 28 vol \%). El caso presentado tuvo valores de hemoglobina de $9,9 \mathrm{~g} / \mathrm{dL}$ antes del episodio hemorrágico y de $8 \mathrm{~g} / \mathrm{dL}$ en el control posterior al procedimiento, con valores de hematocrito de 31 vol \% y 25 vol \%, respectivamente.

En cuanto a los efectos no deseados, Yoong y cols. (4) establecieron que la isquemia uterina puede ser una preocupación, ya que el "sándwich uterino" combina fuerzas de compresión externas con internas del taponamiento, aplicando presión a ambas superficies del miometrio. Para minimizar el riesgo de desvascularización inadvertida en el miometrio, es importante reconocer signos de blanqueo uterino visible y el uso de un volumen de inflado medio inferior de $300 \mathrm{~mL}$ en lugar del límite sugerido de $500 \mathrm{~mL}$. Las pacientes de dicho estudio asistieron a su revisión posnatal a los tres meses y durante esa etapa, habían reanudado la menstruación. Posteriormente, solo 7 pacientes acudieron a seguimiento ecográfico, demostrando normalidad con revestimiento endometrial sin evidencia de sinequias uterinas ni colección de líquido. En la reevaluación de la paciente de este caso, se evidenció retorno del ciclo menstrual y en el control ecográfico a los 6 meses, no se observaron alteraciones.

Todos los reportes revisados en la literatura (4-7) que han descrito la conducta del sándwich uterino para el control de HPP, tienen en común el beneficio adicional del control del sangrado, la estabilidad hemodinámica y la recuperación del tono uterino, con la consecuente conservación del útero.

A propósito del reporte del presente caso, sustentado en la revisión realizada, se muestra una técnica para proporcionar un enfoque menos agresivo en casos de atonía uterina, cuando el tratamiento médico uterotónico disponible ha fallado y el sangrado es persistente, comprometiendo la vida de la paciente. Dos estrategias de tratamiento conservador se combinaron para proporcionar una alternativa exitosa frente a la histerectomía y sus riesgos quirúrgicos asociados en un contexto de carencia de otras alternativas de fármacos uterotónicos, hemostáticos y hemoderivados.

\section{CONCLUSIONES}

Cuando la HPP persiste, a pesar de haber aplicado tratamiento médico y pone en riesgo la vida de la paciente obstétrica, se debe considerar una aplicación secuencial de intervenciones conservadoras basadas en facilidad y rapidez de la terapia. Esto incluye sutura de compresión y/o desvascularización selectiva, seguida del taponamiento uterino con la configuración del sándwich uterino para finalmente realizar una histerectomía en caso de no lograr la hemostasia. Tal enfoque, paso a paso, ayudaría a minimizar la confusión en el quirófano y la decisión apresurada de una histerectomía que pudiera ser evitada tomando en cuenta que este procedimiento aumenta el riesgo de morbimortalidad materna.

La combinación de técnicas no solo participa en la detención del sangrado, sino que ofrece la oportunidad de la estabilización hemodinámica de la paciente y la recuperación del tono uterino, eventos que se conjugan para tener el beneficio de conservar el útero y por lo tanto la fertilidad.

Cada día surgen nuevos retos en la práctica médica en países en los cuales, las carencias de insumos son notables, lo que impone retos para la prevención de complicaciones posparto, además de esfuerzos para alcanzar metas que disminuyan la morbimortalidad materno fetal; de allí que el objetivo primario de esta conducta es proporcionar los principios basados en evidencia para el desarrollo estratégico de conductas que salven vidas, permitiendo una rápida y efectiva 
respuesta ante la emergencia y sugerir intervenciones eficaces para reducir la carga global que representa la HPP.

\section{REFERENCIAS}

1. Federación Argentina de Sociedades de Ginecología y Obstetricia FASGO [Internet]. Buenos Aires: Actualización de Consenso de Obstetricia FASGO 2019 "Hemorragia postparto"; 2019 [consultado el 5 de marzo de 2021]. Disponible en: http://www.fasgo.org. ar/archivos/consensos/Consenso_2019_Hemorragia_ Post Parto.pdf

2. Watkins EJ, Stem K. Postpartum hemorrhage. JAAPA. 2020; 33(4):29-33. doi: 10.1097/01. JAA.0000657164.11635.93.

3. Rodríguez J, Veroes J, González L, González M. Uso del balón SOS Bakri en atonía uterina. Serie de casos en un hospital universitario de tercer nivel. Rev Obstet Ginecol Venez [Internet]. 2013 [consultado 26 de julio de 2020]; 73(2):88-98. Disponible en: http://www.sogvzla. org.ve/sogvzla $20186 / \mathrm{cms} / \mathrm{svcobtenerpdfrevista.}$ php?id $=0000000067 \&$ tipo $=$ normal \& fila $=4$

4. Yoong W, Ridout A, Memtsa M, Stavroulis A, ArefAdib M, Ramsay-Marcelle Z, et al. Application of uterine compression suture in association with intrauterine balloon tamponade ('uterine sandwich') for postpartum hemorrhage. Acta Obstet Gynecol Scand. 2012; 91(1):147-51. doi: 10.1111/j.16000412.2011.01153.x.
5. Nelson WL, O’Brien JM. The uterine sandwich for persistent uterine atony: combining the B-Lynch compression suture and an intrauterine Bakri balloon. Am J Obstet Gynecol. 2007; 196(5):e9-10. doi: 10.1016/j.ajog.2006.10.887.

6. Matsubara S, Kuwata T, Baba Y, Usui R, Suzuki H, Takahashi $\mathrm{H}$, et al. A novel 'uterine sandwich' for haemorrhage at caesarean section for placenta praevia. Aust N Z J Obstet Gynaecol. 2014; 54(3):283-6. doi: 10.1111/ajo.12184.

7. Price N, Whitelaw N, B-Lynch C. Application of the B-Lynch brace suture with associated intrauterine balloon catheter for massive haemorrhage due to placenta accreta following a second-trimester miscarriage. J Obstet Gynaecol. 2006; 26(3):267-8. doi: 10.1080/01443610600555634.
Recibido 5 de agosto de 2020 Aprobado 3 de noviembre de 2020 